\title{
Retropharyngeal Foreign Body
}

\author{
Lt Col AK Mehta*, Col SS Panwar ${ }^{+}$, Surg Cdr RK Verma ${ }^{\#}$
}

MJAFI 2004; 60 : 390-391

Key Words : Foreign body; Retropharynx

\section{Introduction}

$\mathrm{H}_{\mathrm{u}}^{\mathrm{y}}$ ypopharyngeal and oesophageal foreign bodies are usually intraluminal but sharp foreign bodies can get impacted submucosally or intramurally due to inadvertent attempts at removal by the patient or by the inexperienced surgeon. The present case depicts an unusual presentation of a metallic foreign body in the retropharyngeal space without any history of external cervical trauma. To our knowledge such a case has not been reported in literature.

\section{Case Report}

A 45 year old serving junior commissioned officer (JCO) presented with complaints of foreign body sensation in the throat of 3 days duration. He gave history of ingesting a foreign body while having a chicken meal. He felt a sudden sharp pain in his throat followed by persistent foreign body sensation. He was immediately referred for ENT consultation. His only symptom was foreign body sensation in the throat. There were no complaints of regurgitation, vomiting, hemoptysis or haematemesis. There was no dysphonia, dyspnoea or stridor. On examination his general condition was satisfactory. Vital parameters were well maintained. Throat examination was normal. Indirect laryngoscopy did not reveal any pooling of saliva, foreign body, oedema or any congestion. Laryngeal inlet and larynx were normal. Neck movements were painless. There was no sign of trauma, swelling or tenderness and laryngeal crepitus was present. Aural and nasal examination was normal. Systemic examination was essentially normal. Patient was investigated. Radiological examination of neck showed a thin foreign body $5 \mathrm{~cm}$ in length lying in a horizontal plane at level of C5 vertebra. There was no widening of prevertebral soft tissue and no air fluid level (Fig 1). CT scan done revealed a metallic density $8 \mathrm{~mm} \times 4 \mathrm{~mm}$ foreign body in retropharyngeal region at C5 vertebral level without any evidence of abscess or cellulitis (Fig 2). Patient was taken up for endoscopy under GA to look for any intraluminal foreign body. Hypopharyngoscopy and oesophagoscopy were normal and no foreign body was detected. There were no signs of internal trauma or any perforating wound over the posterior pharyngeal wall.Palpation of posterior pharyngeal wall did not reveal any abnormality. He was diagnosed as a case of retropharyngeal foreign body. Patient was put on broad spectrum antibiotics. His symptoms of foreign body sensation disappeared in a few days and he became totally asymptomatic. Since he had no dysphagia and there were no features of retropharyngeal abscess or cellulitis no active intervention was done. Patient was discharged and called for review after 3 months. During review he was asymptomatic and had no features of deep neck space infection. Despite the potential severity of the lesion, since the patient had a favourable outcome and was asymptomatic, no surgical approach was considered necessary to retrieve the foreign body.

\section{Discussion}

Foreign bodies in the aerodigestive tract are common in children, geriatric age group and in psychogenic patients. The commonest foreign bodies in the oesophagus are coins and bones.

Oesophageal and hypopharyngeal foreign bodies are usually found intraluminally. There have been very few interesting reports of pharyngeal and oesophageal foreign bodies at sites other than the lumen. Ramdas et al [1] reported a case of a migrating intramural foreign body in the oesophagus. The patient gave history of a foreign body in the aerodigestive tract and radiologically an open safety pin was found lying in the upper oesophagus. Endoscopy was done and was found to be normal. Use of ' $\mathrm{C}$ ' arm image intensifier located the exact position of the foreign body which was found to be intramural at level of C-6/7 cervical vertebrae. The foreign body was removed by external cervical approach.

In the present case too, no foreign body was detected on endoscopy and palpation of posterior pharyngeal wall was also normal. It was thus concluded that the foreign body was retropharyngeal. This was confirmed

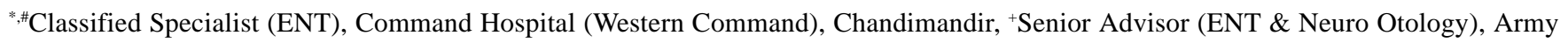
Hospital (R \& R), Delhi Cantt.

Received : 29.7.2002; Accepted : 27.2.2003. 


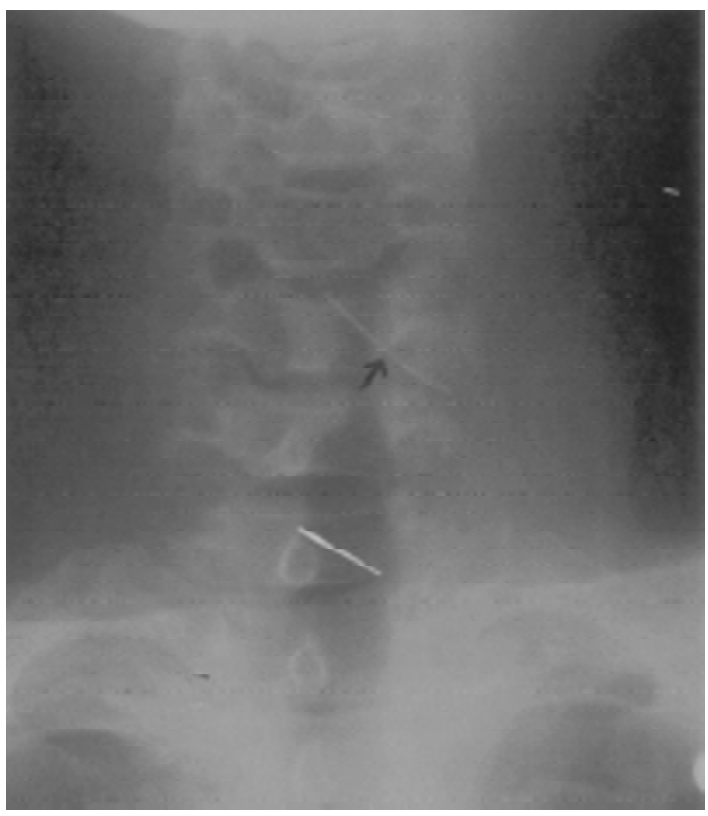

Fig. 1 : Showing metallic foreign body on plain x-ray

radiologically and by $\mathrm{CT}$ scan.

Beyer et al [2] reported a case of a retropharyngeal abscess caused by traumatic perforation of hypopharynx by a fish bone. Endoscopy in their case showed a bulge of the posterior pharyngeal wall. No foreign body was found. A cervical approach was made to drain the abscess and the foreign body was palpated and removed from the retropharyngeal area. Our patient did not reveal any abnormality in the hypophryngeal or oesophageal area. There was no evidence of any perforating injury in the hypopharynx nor any evidence of any abscess.

Other unusual cases of hypopharyngeal foreign bodies have also been reported by Mishra et al [3] who reported an impacted metallic foreign body in left lateral pharyngeal wall just above the level of the epiglottis. The route of entry was external trauma in region of left mastoid process. Though the foreign body impaction was of long standing, there was no neck abscess, emphysema, mediastinitis, internal carotid artery thrombosis or any neuro vascular damage. Jones et al [4] too reported a foreign body penetrating from the ear resulting in a neck abscess.

The nearest similar case to the present one was

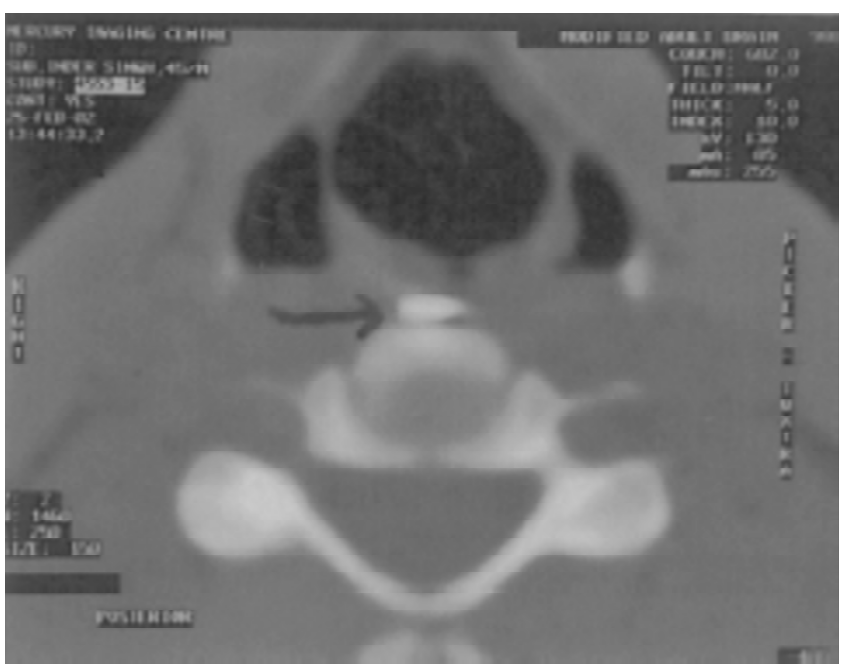

Fig. 2: Showing radioopaque metallic density foreign body in region of C5 vertebra

reported by Pinto et al [5] wherein a 28 year old male patient suffered a penetrating trauma in his neck by a piece of wire. The foreign body lay in the retropharyngeal space at the level of C- 6 vertebra. There were no complications and the patient was managed conservatively with antibiotics and without any surgical intervention.

The present case was unusual in many ways. There was no external trauma nor any sign of local internal injury. Only the history was suggestive of the fact that the foreign body had reached the retropharyngeal space after intraluminal penetration. There were no complications and hence the patient was managed conservatively.

\section{References}

1. Ramdas. Intramural migrating foreign body oesophagus. Ind $\mathbf{J}$ Laryngol Otol Head \& Neck Surg 1995;47:217-8.

2. Bayer S, Eldon J, Gay I. Retropharyngeal Abscess caused by Traumatic Perforation by fish bone. Ann Otol Rhinolaryngol 1990;99:927-8.

3. Mishra A, Shukla G, Bhatia N. Oropharyngeal foreign body. J Laryngol Otol 2000;114:469-70.

4. Jones. Parapharyngeal abscess secondary to external auditory meatus foreign body. J Laryngol Otol 1997;111:1086-7.

5. Pinto. Cervical perforating foreign body: A case report. Rev Assoc Med Bras 2000;46:77-80. 